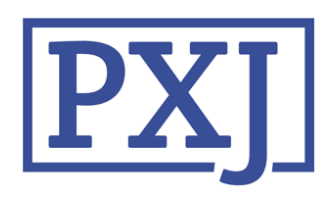

Patient Experience Journal

Volume 4 | Issue 1

Article 11

2017

\title{
Leadership development practices and patient satisfaction: An exploratory study of select U.S. Academic medical centers
}

\author{
Chien-Ching Li \\ Rush University \\ Peter Barth \\ Rush University \\ Andrew N. Garman \\ Rush University \\ Matthew M. Anderson \\ Rush University \\ Peter W. Butler \\ Rush University
}

Follow this and additional works at: https://pxjournal.org/journal

Part of the Health and Medical Administration Commons, Health Services Administration Commons, Health Services Research Commons, Industrial and Organizational Psychology Commons, and the Training and Development Commons

\section{Recommended Citation}

Li C, Barth P, Garman AN, Anderson MM, Butler PW. Leadership development practices and patient satisfaction: An exploratory study of select U.S. Academic medical centers. Patient Experience Journal. 2017; 4(1):97-102. doi: 10.35680/2372-0247.1123.

This Research is brought to you for free and open access by Patient Experience Journal. It has been accepted for inclusion in Patient Experience Journal by an authorized editor of Patient Experience Journal. 
Leadership development practices and patient satisfaction: An exploratory study of select U.S. Academic medical centers

\section{Cover Page Footnote}

The research protocol used in this study was reviewed and approved by the Institutional Review Board of Rush University. The authors wish to thank the National Center for Healthcare Leadership for providing access to the leadership survey data used in this research. 


\title{
Leadership development practices and patient satisfaction: An exploratory study of select U.S. Academic medical centers Chien-Ching Li, Rush_University, Chien-Ching_Li@rush.edu \\ Peter Barth, Rush University,Peter.Barth@navigant.com \\ Andrew N. Garman, Rush University, andy_n_garman@rush.edu \\ Matthew M. Anderson, Rush University, matthew_m_anderson@rush.edu \\ Peter W. Butler, Rush University, Peter_Butler@rush.edu
}

\begin{abstract}
Interest has been growing among academic medical centers (AMCs) in organization-wide strategies that may improve patient satisfaction. Although leadership development programs have been cited as a potentially useful approach, thus far almost all evidence has come from single-organization case studies. The present study sought to examine potential relationships between leadership development and patient experience across organizations. Data for leadership development practices were obtained from a survey conducted by the National Center for Healthcare Leadership. Patient experience data were obtained from the U.S. Hospital Consumer Assessment of Healthcare Providers and Systems (HCAHPS). Multivariate analyses (general linear regressions) were performed to examine the influences of leadership development practice on HCAHPS patient satisfaction scores after controlling for organization characteristics. A total of 23 AMCs met criteria for the study. Multivariate regression analyses identified statistically significant relationships between patient satisfaction scores and three leadership development dimensions: incorporating administrative fellowships, strategically aligning leadership development, and the overall composite score. Findings provide preliminary evidence that leadership development practices may be another useful strategy for improving patient experience outcomes. Future studies involving larger samples are needed to determine how generalizable these findings may be, as well as which specific leadership development practices may be most impactful. This is the only study we are aware of that links leadership development practices to patient experience outcomes at the organization level.
\end{abstract}

\section{Keywords}

Evidence-based practice, leadership development, HCAHPS, human resources, patient satisfaction

\section{Note}

The research protocol used in this study was reviewed and approved by the Institutional Review Board of Rush University. The authors wish to thank the National Center for Healthcare Leadership for providing access to the leadership survey data used in this research.

\section{Introduction}

Attention to improving the patient experience on the part of United States healthcare executives has been growing rapidly in recent years, in part because of the influence of value-based purchasing. Affordable Care Act (ACA) legislation sought to address escalating cost and quality concerns by, among other changes, implementing pay-forperformance incentives ${ }^{1,2}$. Consequently, an increasing proportion of reimbursement is tied to patient outcome measures, including the patient experience ${ }^{3}$. Beyond reimbursement considerations, consumers are also increasingly empowered to access and understand information about patient experience. Mandatory Hospital Consumer Assessment of Healthcare Providers and Systems (HCAHPS) surveys provide publicly available data about patients' experiences with their hospital care in ways that allow these hospitals to be compared directly.

This public availability has also allowed researchers to investigate organizational differences, and identify organizations that have been particularly successful in improving the patient experiences. Prior research has found that Academic Medical Centers (AMCs), as a group, tend to have less favorable patient experiences than their non-AMC counterparts ${ }^{4}$.

These differences could be due to a combination of factors, including their relatively large size and the complexity of the care they provide. This complexity could make service consistency much more difficult to achieve, requiring far more in the way of systematic 
training and monitoring. Case studies of successful performance improvements within AMCs seem to support this possibility, in that they frequently cite the importance of leadership development in successful patient experience initiatives. For example, the Cleveland Clinic's patient experience transformation included implementing an ongoing program of quarterly, daylong, mandatory trainings for their 2,300 managers ${ }^{5}$. UCLA medical center, in describing their journey to patient experience excellence, cite numerous leadership development practices as cornerstones of their success, including their systematic approaches to hiring and talent reviews in addition to systematic service training and monitoring ${ }^{6}-$ approaches that were later successfully replicated at Stanford ${ }^{7}$.

Thus far, studies of the impact of leadership development practices on patient experience outcomes has been limited to case reports such as those cited above. When examining individual cases, it is very difficult to assess the strength of the association between a given factor, such as a specific leadership practice, and a given outcome, such as improvement in patient experience. Studies involving comparable practices across multiple health systems can begin to overcome this problem, by allowing for statistical measures of association.

The non-profit National Center for Healthcare Leadership (NCHL) provided the opportunity to begin investigating these relationships. NCHL defines leadership development according to a set of eleven organizational practices, each of which has some evidence of effectiveness in increasing the performance of leaders and the people they work with. In 2014, NCHL began a program of periodically collecting survey data on the use of these practices in U.S. hospitals, in order to facilitate broader dissemination of evidence-based practices as well as support the more systematic study of how leadership development can improve health system performance ${ }^{8}$. The initial data collection included 23 academic medical centers, which, while still a small overall number, created the opportunity for the first time to assess potential relationships between leadership development practices and patient experience outcomes. Our focus in the present paper was to assess the relationships between the leadership development practices in the NCHL survey and patient experience outcomes.

\section{Methods}

\section{Study Design and Sample}

The Institutional Review Board of the authors' university reviewed and approved this study's protocol. We obtained information about leadership development practices from surveys conducted in 2014 by the National Center for Healthcare Leadership (NCHL) as part of ongoing monitoring of evidence-based practices in leadership development $^{8}$. NCHL solicits organizations' participation in these survey programs through a combination of list server outreach, trade journal announcements, and leased e-mail listings of health system chief executive and chief operating officers. Survey completion was voluntary; participants were offered a feedback report on how their organizations' practices compare to all survey respondents, and were eligible for national recognition if their practices were considered industry-leading based on their scores. We collected additional organizational demographic data about participating organizations from the American Hospital Directory (AHD), an online aggregator of organizational information from credible secondary sources 9 .

We obtained patient experience data through the Hospital Consumer Assessment of Healthcare Providers and Systems (HCAHPS), which we retrieved from Centers for Medicare and Medicaid Services' (CMS) Hospital Compare, a government website that publicly reports HCAHPS survey results for all hospitals in the United States ${ }^{10}$. We retained as our study sample the health systems who were listed as members of the Association of American Medical Colleges Council of Teaching Hospitals (COTH) at the time of the study, and who publicly reported their HCAHPS outcome measures. A total of 23 health systems met both of these criteria.

\section{Measures}

Our dependent variable in this study was the HCAHPS patient satisfaction score associated with value-based purchasing, which we calculated by adding the total percentage of patients rating an AMC a 9 or 10 on the eight individual HCAHPS measures, which are the scores AHRQ considers "top box" or most favorable scores. The measures include patient satisfaction with respect to: (1) questions regarding communication with nurses, (2) communication with doctors, (3) staff responsiveness, (4) pain management, (5) communication about medicines, (6) discharge information, (7) cleanliness and quietness of hospital environment, and (8) overall rating of hospital ${ }^{11}$. An AMC can earn a maximum total score of 800 if $100 \%$ of patients ranked the facility at a level of nine or ten in all eight HCAHPS measures $(100 \%$ x 8 categories $=800)$.

We evaluated leadership development practices based on dimensions and composite scores from the 2014 NCHL Survey ${ }^{8}$. The survey included eleven dimensions, each of which is standardized to a 0-100 scale based on the level and sophistication of practices associated with the dimension. Individual dimensions are also used to calculate a composite score as follows: (1) Strategically aligning leadership development $(20 \%)$, (2) Attracting and selecting leaders $(10 \%)$, (3) Providing developmental experiences $(10 \%)$, (4) Providing performance feedback (10\%), (5) Proactively planning for continuity and future needs $(10 \%)$, (6) Developing clinical leadership strength $(10 \%),(7)$ Monitoring and achieving results (10\%), (8) 
Table 1: Relationships between organizational characteristics and HCAHPS scores $(\mathbf{N}=23)$

\begin{tabular}{lccc}
\hline & & \multicolumn{2}{c}{ HCAHPS Patient Satisfaction Score } \\
\cline { 3 - 4 } & $\mathrm{N}(\%)$ & Mean (SD) & P-value \\
\hline Hospital Region & $7(30.4 \%)$ & $538.3(23.8)$ & \\
$\quad$ Northeast & $7(30.4 \%)$ & $546.9(34.4)$ & \\
$\quad$ Midwest & $5(21.7 \%)$ & $538.6(29.9)$ & \\
$\quad$ West & $4(17.4 \%)$ & $563.3(13.1)$ & \\
$\quad$ South & & & \\
& & & \\
Hospital Size (\# of Beds) & $4(17.4 \%)$ & $530.8(34.7)$ & \\
$\quad<500$ & $19(82.6 \%)$ & $548.4(27.4)$ & \\
$>500$ & & & \\
& & & \\
Ownership Status & $18(78.3 \%)$ & $547.4(24.9)$ & \\
$\quad$ Not-for-profit & $5(21.7 \%)$ & $537.8(37.3)$ & \\
$\quad$ Public & &
\end{tabular}

Preparing new leaders for success (5\%), (9) Identifying and developing high potentials $(5 \%),(10)$ Developing for diversity and inclusion (5\%), and (11) Incorporating administrative fellowships (5\%). A copy of the survey is available on the NCHL website or by request from corresponding author.

\section{Statistical analysis}

We performed bivariate analyses to examine relationships hospital characteristics and leadership practice on HCAHPS patient satisfaction scores using Mann-Whitney $\mathrm{U}$ test and Kruskal-Wallis test for categorical variables, and Spearman correlation test for continuous variables. Additionally, we conducted general linear regression analyses to control for the following organizational demographics that have previously been shown to have an association with HCAHPs scores: organization size (number of staffed beds), region (Northeast, Midwest, West and South), and ownership status (non-governmental not-for-profit and public organizations such as federal, state or local government agency or institutions ${ }^{12}$. SPSS version 18 was used for all statistical analyses.

\section{Results}

As shown in Table 1, the majority of AMCs in our study had bed sizes over 500 (82.6\%), were not-for-profit organizations $(78.3 \%)$, and were located in the West or Midwest (52.1\%). We did not find statistically significant differences in patient satisfaction scores associated with these organization demographics. The mean composite AMC leadership development score was $39.6(S D=19.7)$ and the subcategory with the highest mean score was strategically aligning leadership development, with a mean score of $57.7(S D=26.6)$ (Table 2$)$.
Our bivariate analyses (Table 2) found positive relationships between HCAHPS scores and leadership development practices, however the relationships were only statistically significant for three of the 11 dimensions as well as the leadership development Composite score $(r$ $=0.44, p=0.034)$. The three dimensions with statistically significant associations were: Strategically aligning leadership development $(r=0.42, p=0.045)$, Preparing new leaders for success $(r=0.59, p=0.003)$, and Incorporating administrative fellowships $(r=0.44, P=$ $0.038)$.

Our multivariate analyses (Table 3) which controlled for hospital characteristics (region, bed size and ownership status) found that the relationship between HCAHPS and Composite AMC leadership development scores remained significant $(b=0.68, p=0.038)$. The relationships also remained significant for Preparing new leaders for success $(b=0.41, p=0.014)$ and Incorporating administrative fellowships $(b=0.40, p=0.015)$. In terms of magnitude of these effects, a 10-point increase in composite leadership development scores, preparing new leaders for success, and incorporating administrative fellowships was associated with a correspondingly higher HCAHPS scores of $6.8,4.1$, and 4.0 points, respectively.

\section{Discussion}

Our findings provide preliminary evidence of a relationship between AMC leadership development and HCAHPS patient satisfaction scores. Although the statistical power of our analyses is limited due to the small sample size, the effect sizes appear highly promising, and suggest future study is warranted. In the AMCs studied, every 10.0-point increase in the composite leadership development score was associated with a 6.8-point 
Table 2: Associations between leadership development practices and HCAHPS score: Bivariate Analysis

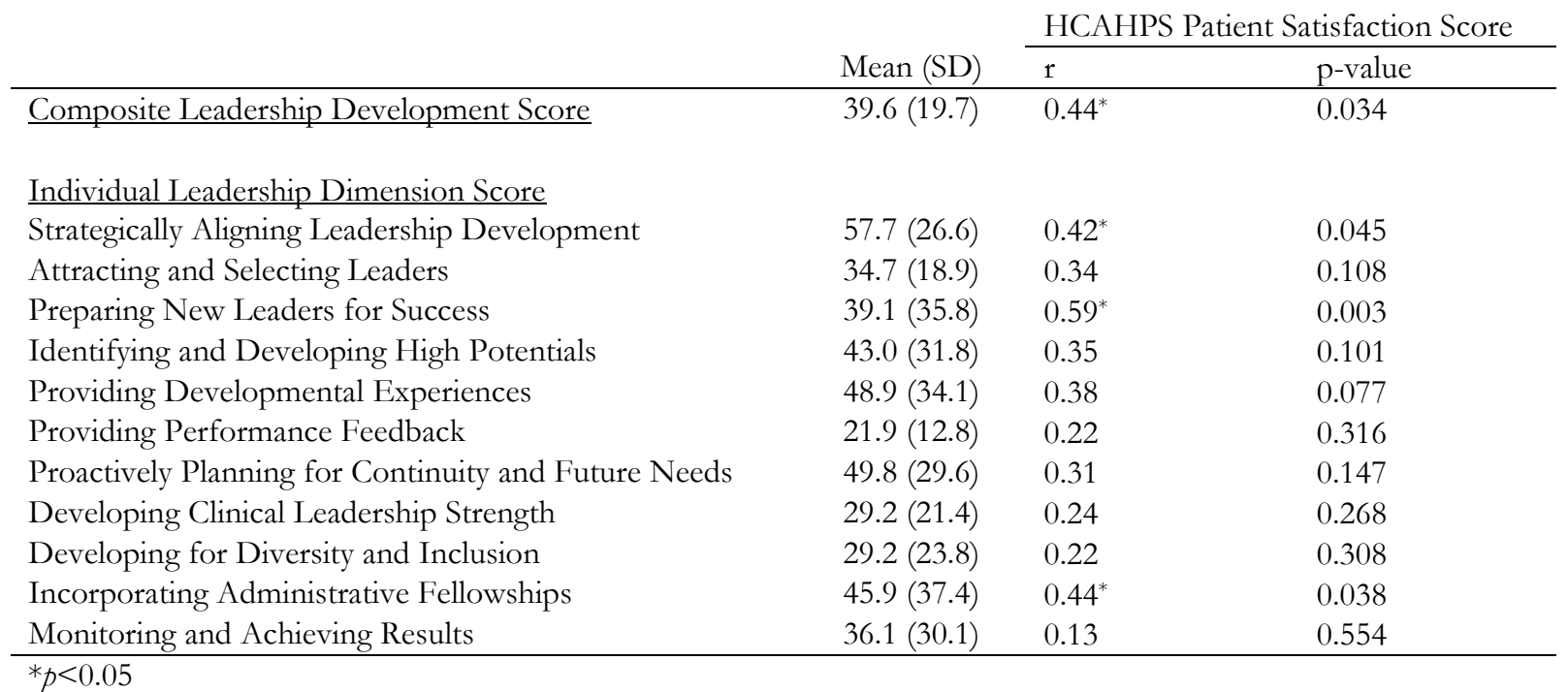

Table 3: Associations between leadership development practices and HCAHPS score: Multivariate Analysis

\begin{tabular}{|c|c|c|c|}
\hline & \multicolumn{3}{|c|}{ HCAHPS Patient Satisfaction Score } \\
\hline & B & S.E. & p-value \\
\hline Composite Leadership Development Score & $0.68^{*}$ & 0.30 & 0.038 \\
\hline \multicolumn{4}{|l|}{ Individual Leadership Dimension Score } \\
\hline Strategically Aligning Leadership Development & 0.43 & 0.25 & 0.099 \\
\hline Attracting and Selecting Leaders & 0.32 & 0.38 & 0.413 \\
\hline Preparing New Leaders for Success & $0.41 *$ & 0.15 & 0.014 \\
\hline Identifying and Developing High Potentials & 0.26 & 0.20 & 0.203 \\
\hline Providing Developmental Experiences & 0.32 & 0.20 & 0.131 \\
\hline Providing Performance Feedback & 0.26 & 0.49 & 0.602 \\
\hline Proactively Planning for Continuity and Future Needs & 0.29 & 0.22 & 0.205 \\
\hline Developing Clinical Leadership Strength & 0.37 & 0.32 & 0.268 \\
\hline Developing for Diversity and Inclusion & 0.33 & 0.32 & 0.315 \\
\hline Incorporating Administrative Fellowships & $0.40^{*}$ & 0.15 & 0.015 \\
\hline Monitoring and Achieving Results & 0.25 & 0.26 & 0.350 \\
\hline
\end{tabular}

Each model controlled for hospital region, hospital size, and ownership status. $* p<0.05$

increase in the HCAHPS VBPP score. Results also suggested that certain types of leadership development activities, including strategic alignment, provision of administrative fellowship programs, and preparing new leaders for success, had particularly strong associations with HCAHPS scores.

In considering the potential implications of these findings, there are several important limitations to that need to be kept in mind. Foremost among these is the size and representativeness of the study sample. While the substantial effect sizes we found gave us greater confidence that the relationships between leadership development practices and HCAHPS outcomes were relatively robust, with such a small sample the risks are also greater that the results were affected by characteristics unique to the organizations under study. A second limitation relates to the voluntary nature of survey participation, with the primary enticements being the opportunity for benchmarking and possible recognition 
for exemplary practices. Given this recruiting orientation, survey respondents may have been more oriented toward leadership development as a core organizational competency than non-respondents, and thus represent a restricted range. A third limitation relates to the self-report nature of the leadership practices survey. Although items were designed to be as objectively measured as possible, there were few safeguards to ensure accurate interpretation and application. Lastly, the association between leadership development practices and HCAHPS outcomes does not establish causation. Future studies could usefully expand on the present work by expanding the size of the sample, examining changes in HCAHPS scores over time, or both.

These limitations notwithstanding, our results provide at least preliminary quantitative evidence that leadership development practices may be an important strategy to consider in improving value based purchasing

disbursements. The three dimensions identified as holding the strongest associations with HCAHPS scores may each affect these outcomes in different ways. For example, Strategically aligning leadership programs, according to NCHL's definition, involves organizations tying their programs explicitly to the strategic objectives of the organizations, as well as senior leaders playing an active role in their ongoing operation ${ }^{8}$. Assuming HCAHPS scores were expressed as corporate objectives by the organizations in this study, tying leadership development activities explicitly to these objectives should translate into greater impact on improving their scores. Alternatively, leadership development practices associated with Preparing new leaders for success may affect HCAHPS scores indirectly, through their association with higher levels of job performance and staff retention ${ }^{15}$. Specific practices such as 30,60 and 90 day check-ins, combined with planned approaches to socialization and job coaching, are examples of effective components of this dimension ${ }^{13}$.

Finally, Incorporating administrative fellowships may also affect HCAHPS scores through several additional mechanisms. Administrative fellowships typically involve one to two- year postgraduate roles created to attract new, high-potential talent into positions that provide increasing leadership responsibilities and guidance. Fellowship programs could be associated with higher HCAHPs scores by creating greater slack capacity for pursuing HCAHPsrelated process improvements, a greater relative emphasis on development of healthcare management profession, and/or a broader strategy for attracting cultivating highpotential management talent. With respect to the latter, previous research suggests that administrative fellowships tend to attract higher quality talent pools than other entrylevel administrative roles ${ }^{14}$, and as a result these programs create the potential for strengthening administrative leadership over time.

\section{Conclusion}

Although links between clinical outcomes and healthcare leadership in AMCs have been actively studied ${ }^{15,16}$, research that can guide AMCs' efforts to improve patients' experience of care by improving leadership are in their infancy. Through the present study, we sought to make an initial contribution to begin filling this research gap, and to encourage other scholars to begin investigating which approaches to leadership development seem to hold the most promise for improving the patient experience as well as other patient-related outcomes.

\section{References}

1. Gregory D, Curfman MD, Morrissey S, Drazen JM, High-value health care: A sustainable proposition. $N$ Engl J Med. 2013; 369:1163-1164

2. Porter EM, What is value in health care? New England Journal of Medicine 2010; 363:2477-2481

3. Manary MP, Boulding W, Staelin R, Glickman S, The patient experience and health outcomes. $N$ Engl J Med. 2013; 368:201-203

4. Shahian DM, Nordberg P, Meye GS, et al. Contemporary performance of U.S. teaching and nonteaching hospitals. Acad Med. 2012; 87(6):701-708.

5. Merlino JL, Raman A, Health care's service fanatics. Harvard Business Review. May 2013; 91(5): 108-116.

6. Michelli JA. Prescription for Excellence: Leadership lessons for creating a world-class customer experience from UCLA Health System. New York: McGraw-Hill; 2011.

7. Rubin A, Prigge T. Rapid culture change in an Academic Medical Center: This is not an oxymoron. National Center for Healthcare Leadership web site. https://nchlblog.org/2013/01/14/rapid-culturechange-in-an-amc-this-is-not-an-oxymoron-withstanfords-amir-rubin-and-todd-prigge/ Published January 14, 2013.

8. Anderson M, Garman A. Leadership development in healthcare systems: Toward an evidence-based approach. National Center for Healthcare Leadership. http://nchl.org/Documents/Ctrl_Hyperlink/NCHL _Leadership_Survey_White_Paper_Final_05.14_uid6 232014300422.pdf. Accessed April 24, 2015.

9. American Hospital Directory. Information about hospitals from public and private data sources including MedPAR, OPPS, hospital cost reports, and other CMS files. (n.d.). Accessed March 8, 2015.

10. Centers for Medicare \& Medicaid Services. HCAHPS: Patients' perspectives of care survey. http://www.cms.gov/Medicare/Quality-InitiativesPatient-AssessmentInstruments/HospitalQualityInits/HospitalHCAHPS. html. Accessed November 21, 2014.

11. Hospital Consumer Assessment of Healthcare Providers and Payment Systems. A step-by-step guide to calculating the patient experience of care domain score in the hospital value-based purchasing FY 2013 actual percentage payment summary report. 
https://www.hcahpsonline.org $\% 2$ FFiles $\% 2$ FHospital $\% 2520$ VBP $\% 2520$ Domain $\% 2520$ Score $\% 2520$ Calcula tion $\% 2520$ Step-by-

Step \%2520Guide_V2.pdf\&usg=AFQjCNFsrLUETvAIZzlpY4osZZI69WGyg\&sig2=vaVIqLPwuWP1_ QTCsQyv7w\&bvm=bv.108194040,d.cWw Accessed March 6, 2015.

12. Jha A, Orav E, Zheng J, Epstein A. Patients'

Perception of Hospital Care In The United States. N Engl J Med. 2008; 359(18):1921-1931.

13. Bauer, T. Onboarding new employees: Maximizing success. Society for Human Resource Management 2010.

https://www.shrm.org/about/foundation/products/ Pages/OnboardingEPG.aspx. Accessed May 25, 2015.

14. National Council on Administrative Fellowships. http://nchl.org/static.asp?path $=2851,6549$. Accessed May 25, 2015.

15. Keroack M, Youngberg B, Cerese J, Krsek C, Prellwitz L, Trevelyan E. Organizational factors associated with high performance in quality and safety in academic medical centers. Acad Med. 2007; 82: 1178-1186.

16. Wong C, Cummings G. The relationship between nursing leadership and patient outcomes: A systematic review. J Nurs Manag. 2007; 15: 508-521. 